\title{
EL NOVUM EN LA CIENCIA FICCIÓN COSTARRICENSE
}

\author{
Roy Alfaro Vargas
}

\section{(c)}

Esta obra está bajo una licencia Creative Commons

Reconocimiento-No Comercial-Sin Obra Derivada 



\title{
EL NOVUM EN LA CIENCIA FICCIÓN COSTARRICENSE
}

\author{
THE NOVUM IN COSTA RICAN SCIENCE FICTION
}

Roy Alfaro Vargas

\begin{abstract}
RESUMEN
Este artículo analiza la así llamada ciencia ficción costarricense, en relación con el concepto de novum desarrollado por Darko Suvin. Se plantea la poética suviniana como referente para el análisis y construcción del género de ciencia ficción, en cuanto tal enfoque permite la elaboración de una ciencia ficción que aglutine el contexto empírico del escritor y las posibilidades ocultas tras tal contexto. Asimismo, se define la ciencia ficción como una literatura crítica, que en nuestra actual situación implica una crítica a la nomocracia posmoderna. De igual modo, se establece que la denominada ciencia ficción costarricense no es realmente ciencia ficción.

Palabras clave: Literatura costarricense, cuento, ciencia, posmodernidad, neoliberalismo.
\end{abstract}

\begin{abstract}
This article analyzes the so-called Costa Rican science fiction in relation to the concept of novum developed by Darko Suvin. It is set up the Suvinian poetics as a referent for the analysis and construction of the genre of science fiction, as long as such approach allows the elaboration of a science fiction that comes together the empirical context of the writer and the possibilities hidden behind this context. Also, it is defined the science fiction as a critical literature, which in our current situation implies a critique of the postmodern nomocracy. In the same way, it is established that the so-called Costa Rican science fiction is not really science fiction.
\end{abstract}

Key words: Costa Rican Literature, Tale, Science, Postmodernity, Neoliberalism.

\section{Introducción}

La ciencia ficción (CF) es un género de reciente aparición en el contexto de la literatura costarricense. Como toda novedad, esta denominada CF no se ha presentado libre de problemas ligados tanto a su producción por parte de los escritores hoy catalogados dentro de este ámbito, como del lado de la crítica literaria que se enfrenta sin herramientas teóricas pertinentes a este nuevo género. Este artículo pretende llenar ambos espacios, ofreciendo, de manera sucinta, una perspectiva teórico-poética que sea útil al escritor, así como al crítico

Bach. Roy Alfaro Vargas. Investigador independiente. Egresado del Posgrado en Literatura Latinoamericana de la Universidad de Costa Rica. Costa Rica.

Correo electrónico: royalfarov@gmail.com

Recepción: 24- 01- 2014

Aceptación: 15- 03- 2014 
literario. Para esto, se recurrirá a las nociones esbozadas por Darko Suvin (1979), en su libro Metamorphoses of Science Fiction, y quien hoy es reconocido como uno de los principales teóricos en lo que respecta a la $\mathrm{CF}$.

De hecho, este artículo hace una amalgama de las posiciones de Suvin (1979) y de sus continuadores, y cuya síntesis se plantea aquí para darle un fundamento científico y académico al trabajo del crítico literario.

La tesis que guía este trabajo es la siguiente: con base en la perspectiva teórica adoptada, la actual producción literaria costarricense catalogada como CF, no es tal; en cuanto no se logra representar, crear, novum alguno.

Para probar esta tesis, se seguirán los pasos que a continuación se describen: establecer un marco teórico referente al planteamiento suviniano, determinar los factores contextuales (literarios y extraliterarios) que enmarcan la producción y crítica de la CF costarricense, y analizar una muestra amplia de la producción literaria de autores como: Iván Molina, Jessica Clark, Daniel Garro, etc., que permita ejemplificar lo argumentado.

\section{La CF y el novum}

Suvin define la CF de la siguiente manera: "La CF es, entonces, un género literario cuyas condiciones necesarias y suficientes son la presencia e interacción de alienación (estrangement) y cognición, y cuyo recurso formal fundamental es una estructura imaginativa alternativa al ambiente empírico del autor" (Suvin, 1979, p. 7-8). ${ }^{1}$

Así, “'La cognición', con sus implicaciones lógicas, racionales, refiere al aspecto de la $\mathrm{CF}$ que nos da pie para entender, comprender [...]. 'La alienación' [...] refiere a aquel elemento de la CF que reconocemos como diferente, que nos 'extraña' con respecto a lo familiar y cotidiano" (Roberts, 2006a, p. 8). Esta interrelación entre la cognición y la alienación es lo que define el novum.

Visto de este modo, "el novum es una categoría mediadora cuya potencia explicativa surge de su raro entronque entre los dominios literarios y extraliterarios, ficticios y empíricos, formales e ideológicos, en breve de su inalienable historicidad" (Suvin, 1979, p. 64). En este marco, "La CF es tanto lo diferente como lo mismo" (Roberts, 2006a, p. 10), a la vez que "imagina el cambio en términos de la especie humana entera y estos cambios son a menudo el resultado de descubrimientos científicos e invenciones que son aplicados por los seres humanos a su propia evolución social" (Csicsery-Ronay, 2003, p. 113).

Efectivamente, dentro de esta perspectiva, “'La alienación' [...] significa reconocer el novum; 'la cognición' significa evaluarlo, tratando de entenderlo" (Shippey, 2005, p. 15), lo cual requiere del lector (sea un crítico literario o un lector común) un esfuerzo intelectual extra para la comprensión de la CF, en comparación con otras formas de ficción (Shippey, 2005, p. 11), lo cual también obliga al lector a "romper apriorismos sociales" (Moreno, 2006, p. 78). En cierto sentido, dentro del espectro de la CF, "el novum tiene un efecto revolucionario" (Moylan, 2000, pp. 58-59), que permite "extrapolar una idea o inquietud" (Moreno, 2006, p. 68), "no solo en el sentido de ensayar una gran cantidad de conceptos, posibilidades, dramas intelectuales, etc., sino en su más profundo sentido de estructura textual del mundo, planteando alternativas al mundo" (Roberts, 2006b, p. 12).

Entonces, el proceso de recepción de la CF por parte de los lectores, "pone en marcha sus formaciones ideológicas y hace posible nuevos modos de conocer el mundo" 
(Moylan, 2000, p. 61); "Así la alienación cognitiva necesariamente implica un estado de conocimiento parcial e imperfecto" (Parrinder, 2000, p. 7), en cuanto siempre hay un desfase entre lo real (empírico) de donde parte el relato de CF y lo posible que se liga, como rasgo fundamental, al novum.

El novum dentro de la CF mediatiza lo real y lo posible, de donde "las más importantes distinciones entre el mundo del relato y el mundo del lector derivan" (Csicsery-Ronay, 2003, pp. 118-119). De hecho, la CF "requiere una racionalización física, material, más que una arbitraria o sobrenatural. Este fundamento de la CF en lo material más que en lo sobrenatural deviene uno de sus características clave" (Roberts, 2006a, p. 5). La CF "es un discurso, construido sobre ciertos principios lógicos, que evita la autocontradicción; que es racional más que emocional o instintivo" (Roberts 2006a: 9): "El novum es producto de procesos materiales; produce efectos que pueden ser lógicamente derivados de las causas del novum en el mundo social y material; y es posible en términos de lógica histórica" (Csicsery-Ronay, 2003, p. 119).

La mediación real-posible (empírico-pensable) implica "una epistemología situacional y relacional y no una ontología doctrinariamente creída" (Suvin, 2000, p. 258). Por esto, la CF (su novum) se presenta como "una combinación de narrativa, explicaciones de hechos científicos y predicciones de futuras invenciones" (Westfahl, 2005, p. 191) y, casi con absoluta necesidad, situándose "más allá de la actual ciencia y, por ende, en el futuro" (Klein, 2005, p. 125). En efecto, "la ciencia ficción está estrechamente asociada con el futuro [...] Es sobre todo una literatura de cambio y cambio por definición implica que el presente es percibido en relación con las percepciones del pasado y las expectativas del futuro que moldean aquel presente" (Seed, 2011, p. 97).

La flecha del tiempo, donde se asume la ciencia como continuidad y discontinuidad dentro de la $\mathrm{CF}$, lleva a la ciencia a manifestarse textualmente como tecnología: "La tecnología es un indicador fundamental del cambio en la ciencia ficción" (Seed, 2011, p. 47). En este sentido, "La ciencia ficción [...] es intrínsicamente un género de 'alta-información"” (Shippey, 2005, p. 16), que está "continuamente registrando el impacto continuo de la ciencia en las situaciones humanas" (Slusser, 2005, p. 41).

Así, dentro de la representación de la ciencia y la tecnología, "la ciencia ficción debe mostrar un respeto básico por los principios y leyes de la ciencia” (Westfahl, 2005, p. 187). Esto implica que el escritor de $\mathrm{CF}$ debe poseer un conocimiento básico en física, cosmología, robótica, etc., o dicho de otra forma, la CF no puede ser producto de ocurrencias. El escritor de CF debe ofrecer "explicaciones de hechos científicos y/o pasajes expositivos extensos proveyendo evidencia de un proceso de pensamiento científico en operación" (Westfahl, 2005, p. 187). De lo contrario, tal supuesta CF será "universalmente castigada y deslegitimada como ciencia ficción" (Westfahl, 2005, p. 187).

A modo de síntesis y buscando operacionalizar todo lo dicho anteriormente, diremos que la $\mathrm{CF}$ se caracteriza por los siguientes cinco puntos:

a. Presentación de un mundo no completamente comprendido (el novum de Suvin) (Parrinder, 2000, p. 7).

b. Una crítica de nuestra sociedad, de nuestros presupuestos y una forma de acceder al cambio histórico, a nuestro futuro (la idea de la alienación cognitiva) (James, 2000, p. 21).

c. Un cambio del locus de alienación desde lo geográfico a lo cronológico (James, 2000, p. 30).

d. Un rol fundamental de la tecnología (Seed, 2011, p. 47). ${ }^{2}$ 
e. El empleo de neologismos (Seed, 2011, pp. 48-49). Este último punto funciona como un factor de verosimilización, en relación tanto con el supuesto cambio diacrónico de la lengua dentro del universo representado en el relato de $\mathrm{CF}$, como por la cuestión de darle forma lingüística al novum, en función de la alienación cognitiva.

Todos estos elementos funcionan como un todo, no como partes que se agregan de manera cuantitativa, sino cualitativamente, sinérgicamente.

\section{Contexto socio-histórico y literario de la CF costarricense}

A pesar de los antecedentes que se quieren ofrecer alrededor de la CF costarricense, que la ubican como originada con El problema de Máximo Soto-Hall (Molina-Jiménez, 2010) o con La caída del águila (Ríos-Quesada, 2012) o, incluso, con Alberto Cañas (HaywoodFerreira, 2011); así como con antecedentes más recientes como: Una sombra en el hielo (1995) de Laura Quijano, Diez días de un fin de siglo (2007) de Emilia Macaya, y La miel de los mudos (2003) y La conspiración de las zurdas (2007) de Iván Molina-Jiménez (Ríos-Quesada, 2012; Haywood-Ferreira, 2011); lo cierto es que el fenómeno de la denominada CF costarricense adquiere amplitud, legitimidad y divulgación, a partir de Posibles futuros (2009) de la Editorial de la Universidad Estatal a Distancia (EUNED), en el 2009 y la seguidilla de textos que esta editorial ha lanzado como CF (Objeto no identificado [2011] y Lunas en vez de sombras [2013]).

A partir de Posibles futuros, el Grupo Nación y el mismo Iván Molina-Jiménez, unidos a la labor de la EUNED, se lanzan a la legitimación de los cuentos de Laura Casasa[Núñez], Daniel Garro, Laura Quijano, etc., como autores de CF.

No deja de ser interesante que el auge de la CF se ubique en el 2009, después de la crisis financiera del 2008. Tal crisis que no es financiera sino sistémica (Alfaro-Vargas, 2011) conlleva dos vertientes opuestas en todos los planos.

La primera vertiente está ligada a las manifestaciones ultraconservadoras de la nomocracia posmoderna, la cual se define así:

\footnotetext{
La nomocracia posmoderna es, entonces, una formación social comprendida dentro de los parámetros de una economía que jurídicamente tiende teóricamente, a fomentar las oportunidades dentro del mercado (en tanto que generalizables, sin importar la posibilidad real de hacer concretas tales oportunidades) y la necesidad ideológica de centrarse en lo particular (Alfaro-Vargas, 2012, p. 109).
}

Es decir, la nomocracia posmoderna incluye el discurso neoliberal ligado a la ideología posmoderna, con la cuestión de las identidades, de la diversidad, del giro lingüístico, etc. ${ }^{3}$

Esta primera vertiente, más allá de los evidenciados fracasos del neoliberalismo, sigue atada a este en su fase más antidemocrática, a pesar de que "su persistencia acelerará y profundizará la crisis" (Antonio, 2012, p. 583).

Asimismo, en el campo educativo (la socialización formal), en el ámbito de las grandes masas, de las clases no burguesas, el contenido de la educación apto para estas se reduce a: "leer, escribir y contar" (Friedman, 2010, p. 169); sin olvidar que la actual educación va dirigida a "legitimar una concepción muy diferente y muy limitada de democracia" (Apple, 2013, p. 2), a la vez que, más allá de las celebradas identidades múltiples, el neoliberalismo "abre un espacio para algunas identidades y clausura otras" (Apple, 2013, p. 7).

La segunda vertiente, en el contexto mundial, se viene gestando y hoy en Costa Rica tiene muy poca difusión. Esta vertiente se ancla en una actitud crítica con respecto a 
la primera vertiente. Esta vertiente abandona los presupuestos neoliberales que relegaban la economía política al cesto de la basura: "la crítica de la economía política está retomando su lugar primordial en la teoría y la práctica" (Fraser, 2013, p. 5); lo cual implica para esta feminista (Nancy Fraser) que el feminismo ha fallado, así como los estudios de subalternidad y los estudios culturales que "no pueden ser ni una teoría del capitalismo globalizante ni su crítica" (Chibber, 2013, p. 286). Así, "si una teoría no puede comprender el sentido básico de cómo funciona el capitalismo, entonces la idea de suplantar los radicales análisis marxistas o de otro tipo, no pueden ser tomados en serio" (Chibber, 2013, p. 288).

Como vemos, esta segunda vertiente del contexto socio-económico de la CF costarricense retoma a Marx y al marxismo, a la vez que rompe con el giro lingüístico, al afirmar que: "todo lenguaje es referencial" (Gaskin, 2013, p. 290), rechazando "la idea que el mundo es textual en cualquier sentido literal" (Gaskin, 2013, p. 240). Por tanto, dentro de las corrientes que cuestionan la nomocracia posmoderna, el capitalismo y el giro linguiístico, "el lenguaje poético es un modo de aprehender la realidad" (Gaskin, 2013, p. 290).

Volviendo a la CF costarricense, decíamos que claramente este fenómeno literario está supeditado en su expansión y legitimación al periodo posterior a la crisis del 2008. De igual modo, es un fenómeno ligado a la denominada Promoción de 1980 planteada por QuesadaSoto (2000) y que se caracteriza por sus vínculos con el neoliberalismo y el posmodernismo (Quesada-Soto, 2000, pp. 42-43). Iván Molina ejemplifica, en su cuento "Anti-Eureka” (2010), esto de manera muy clara: "La democracia y la secularización están apenas en ciernes, y la equiparación sostenible de derechos, entre los sexos, las etnias y las naciones, es un proceso que todavía no inicia” (Molina-Jiménez, 2010, p. 22).

Aquí Molina, a través de su personaje Razatroc (Cortázar al revés), miembro de una raza superior que ha alcanzado un punto absoluto de desarrollo (por supuesto el neoliberalismo para Molina), esgrime, para nosotros la raza inferior, parte del credo neoliberal. De igual modo, Molina en "Sputnik" (2009) hace mofa del marxismo, al plantear una trama donde Jennifer (quien vive en el 2107 y viene de un futuro nomocrático posmoderno, de hecho visto como expresión eternizante del capitalismo) viaja al pasado y oye una conferencia de un comunista llamado Víctor Manuel Ríos, quien pronostica que: "Obreros rojos construirían fábricas y ciudades en Marte y cruceros revolucionarios navegarían regularmente entre Mercurio y Plutón" (Molina-Jiménez, 2009, p. 51). El discurso de Víctor Manuel Ríos ya está negado, desde el inicio, por la situación vivencial de donde Jennifer parte. Así el credo neoliberal, el antimarxismo, etc., son elementos presentes en los autores de la CF costarricense, lo cual, repetimos, los ubica dentro de la Promoción de 1980, como un subgrupo tardío de esta promoción, ligado a la decadencia represiva del neoliberalismo y, en efecto, en tanto negación de la segunda vertiente socio-económica que marca el contexto de emergencia de la supuesta CF costarricense.

\section{El novum y la CF costarricense}

En este apartado, analizaremos los cinco puntos dados arriba, como elementos que definen la CF, en relación con algunos textos y autores, como: "Joyería mental" (2013), "Ragnarök" (2012), "Bajagua" (2011) y "Frente frío" (2009) de Jessica Clark. De Iván MolinaJiménez: "Sputnik" (2009), "Sustituto" (2009), "Habitante de los márgenes" (2009), "Morpho" (2009), "Costarricense interino" (2009), "Venus desciende" (2009), "Soy la de la foto" (2009), 
"Xelajú" (2009), "La última noche de Rodolfo" (2009), "Armazón de estrellas" (2009), "Pandora inminente" (2009), "Anti-Eureka" (2010), "Sueños combatidos" (2011), "Marte inesperado" (2012) y "Por tierra firme" (2013). De Laura Quijano: "Flor del crepúsculo" (2009), "El modelo defectuoso" (2010) y “Objeto no identificado” (2011). De Evelyn Ugalde: “A Costa Rica, ¡Nunca más!" (2010) y "Amor virtual" (2011). De Mariana Castillo-Rojas: "En su defensa" (2010) y "Sin protocolos de seguridad" (2011). De Manuel Delgado: "Las campanas" (2010) y "Órdago" (2011). De Antonio Chamu: "La onceava generación" (2009) y "Otro barril de amontillado" (2010). De Luis E. Jara: "El legado del cuervo" (2010). De Daniel Garro: "Vórtice" (2010), "El ejército de Onara" (2011) y "El vuelo final de Kayla 8" (2012). De Ericka Lippi Rojas: "El impulso necesario para despertar" (2012) y "Lunas en vez de sombras" (2013). De David Díaz Arias: "La tropa" (2009) y "Los señores de piedra" (2012). De Laura Casasa-Núñez: "Los túneles de la memoria" (2009). De Alberto Ortíz: "Raquel y los emperadores" (2011). De José Ricardo Chaves: "Yo, la criatura" (2013). De Daniel González Chaves: "Sofía" (2013) y de Ana Cristina Rossi: "Abel” (2013). ${ }^{4}$

Iniciemos con el empleo de neologismos. Existe en la CF costarricense la tendencia a la formación de neologismos, con el simple recurso del uso de prefijos. Por ejemplo: nanocámaras ("Sputnik"), holocuartos ("En su defensa”), poroplástica ("El vuelo final de Kayla 8"), nanosegundos ("Sueños combatidos"), ciberempresas ("Raquel y los emperadores"), etc. Asimismo, se hace uso de términos ya comunes dentro de la $\mathrm{CF}$, por ejemplo: androide ("Lunas en vez de sombras"), robots ("Amor virtual"), cyborgs ("El impulso necesario para despertar"), etc.

Ambas formas de aplicación de neologismos aparecen en la CF costarricense como un mecanismo de verosimilización, mas sin la suficiente elaboración, ya que muchas de las "nuevas" palabras, por su propio mecanismo de construcción morfológica, conllevan un anclaje inevitable con respecto al contexto empírico compartido por el escritor y el lector, lo cual plantea la no-generación de novedad, de extrañamiento. Luego, esta ausencia de extrañamiento se ve incrementada por palabras de uso común y repetitivo dentro de la $\mathrm{CF}$; referimos a términos como robot, cyborg, etc.

De igual manera, el neologismo debe reflejar el desarrollo diacrónico de la lengua en el contexto particular del relato de CF y, además, debe corresponder también a un cambio cualitativo en el desarrollo científico-tecnológico. Sin embargo, algunos cuentos se contentan con simplemente mencionar, por ejemplo, computadoras ("Bajagua", "Amor virtual", etc.) o del todo no incorporan ningún elemento novedoso a nivel del lenguaje. Por ejemplo: "Órdago", "Los señores de piedra", "Ragnarök", "Por tierra firme”, "Abel”, "Sofía”, "Yo, la criatura", "A Costa Rica, „Nunca más!”, "Frente frío”, “La tropa”, Flor del crepúsculo”, etc.

Efectivamente, hay un abuso de clichés en relación con la creación de neologismos dentro de la CF costarricense. No obstante, esto está unido al rol que juega la tecnología dentro del fenómeno costarricense de la llamada CF.

Como mencionábamos en el primer apartado, la cuestión tecnológica dentro de la CF no es algo producto de alguna ocurrencia, sino que debe el escritor esforzarse por respetar las leyes científicas y dar las explicaciones necesarias y suficientes sobre los artefactos y aplicaciones tecnológicas que este nos presenta en el texto. O sea, la tecnología debe estar dentro de la trama y no simplemente esbozada en ella.

La CF costarricense, sin embargo, introduce términos como: computadora portátil ("Frente frío"), computadora ("Marte inesperado"), pantalla ("Sustituto"), etc., que no rompen, 
primero, con el contexto empírico del escritor, ni incorpora estos elementos tecnológicos como recursos determinantes del relato, simplemente están ahí como un recurso más de una pretendida verosimilización, tan fallida como el que apuntábamos al hablar de los neologismos.

No obstante, hay un factor más disfuncional dentro de la CF costarricense, el cual muestra no solo una ausencia de conocimiento en áreas científico-tecnológicas por parte de los escritores analizados, sino además una falta total de elaboración del producto que se vende como relatos de CF. Veamos algunos ejemplos, que nos permitirán probar esta afirmación.

Molina-Jiménez (2013), en "Por tierra firme", vuelve al tópico del viaje en el tiempo planteado ya en "Sputnik" (2009). Así, nos dice en "Por tierra firme": "-Se trata de un procedimiento que permite trasladarse al pasado. Prefiero omitir, por ahora, los detalles técnicos" (Molina-Jiménez, 2013, p. 62).

En boca del personaje, de hecho, Molina-Jiménez (2013) de manera muy evasiva no explica cómo es capaz el procedimiento para trasladarse al pasado y de solventar la cuestión de la segunda ley de la termodinámica (el principio de entropía) que le inserta una unidireccionalidad a la flecha del tiempo (del pasado al presente y de ahí al futuro, nunca a la inversa). La cuestión es clara, el procedimiento apuntado es una llana ocurrencia de Molina-Jiménez (2013), la cual hace evidente un deficiente manejo de las leyes de la física hoy conocidas. Se supone que es un texto de CF (por lo menos así lo legitima la EUNED), pero se queda en una mera fantasía científica.

Sirva también de ejemplo el siguiente texto de Laura Quijano (2010), tomado de "El modelo defectuoso": "un modelo robótico de apariencia vagamente humanoide. Al menos poseían algo similar a una cabeza donde los orificios podían indicar ojos, y una caja rectangular central de la cual surgían cuatro brazos, dos arriba y dos debajo de éstos, y que se apoyaba sobre dos poderosas patas cilíndricas" (Quijano, 2010, 34).

Aquí, la descripción es vaga, poco detallada, superficial, sin imaginación, etc. No hay una visualización por parte de la autora de un aparato tecnológico como el que fallidamente pretende representar, más cuando al final de este cuento nos enteramos que dentro del tal humanoide iba un cerebro humano de contrabando. Con la descripción que da la autora no se le da pie al lector para interpretar y comprender el rol inequívoco de esta tecnología dentro de la trama. A pesar de ser fundamental para el relato, la autora desestimó una adecuada descripción.

La CF costarricense analizada aquí, por ende, no tiene consciencia de la importancia de los recursos científico-tecnológicos dentro de la trama y no como un simple recurso ornamental de verosimilización (finalmente fallida). Además, el buen desarrollo del recurso tecnológico dentro de la trama de la $\mathrm{CF}$, requiere de una crítica literaria que acabe con la idea de que la $\mathrm{CF}$ permanezca como "un objetivo difícil para el examen crítico" (Cramer, 2003, p. 189). En otras palabras, el crítico literario no puede contentarse con calificar de $\mathrm{CF}$, producciones literarias que o son fantasía científica o una mala CF (soft science fiction). El crítico literario también tendrá, entonces, que aprender física, cosmología, etc., para llevar a cabo su función crítica.

En el ámbito del tercer elemento por analizar, el de la crítica social en la CF costarricense, nos enfrentamos con o la inexistencia de ella (sea por omisión o por sacralización del status quo) o por la superficialidad que esta conlleva.

Ya hemos señalado el anclaje adoctrinante de cuentos como "Sputnik" (2009) o “Anti-Eureka” (2010) de Iván Molina-Jiménez, que meramente sacralizan lo dado, la sociedad capitalista y la nomocracia posmoderna. 
Por otra parte, en la gran mayoría de los cuentos leídos para este trabajo, hay una ausencia total de crítica y menos todavía de una crítica ligada a un novum dentro de los imperativos de la CF. De este modo, cuentos como: "El ejército de Onara", "Objeto no identificado", "Ragnarök", "Joyería mental”, "Yo, la criatura", "En su defensa", "Las campanas", "Otro barril de amontillado", etc., muestran la ausencia de una crítica del espacio empírico de donde parte el escritor, quitándole novedad, extrañamiento, a una CF que no articula lo real y lo posible.

En algunos cuentos, se presenta una crítica muy superficial, como en el caso de "Abel", "Amor virtual", "Raquel y los emperadores", "Órdago", "Sin protocolos de seguridad" o "El legado del cuervo". De este último veamos un ejemplo: "Tanto el lector como el perverso cuervo estaban absolutamente sorprendidos con el contenido light de cada párrafo de aquella noticia y de cada una de las páginas del periódico, ilustrado con mujeres de poca ropa y poco pudor" (Jara, 2010, p. 124).

En este extracto del cuento "El legado del cuervo", se pretende criticar los periódicos amarillistas que explotan la imagen femenina. No obstante, dentro de los imperativos del novum de la CF, el texto de Luis E. Jara (2010) no sale de los contornos empíricos del escritorlector, con lo cual se queda en lo real en detrimento de lo posible y, asimismo, no llega a plantear las causas de tal clase de periodismo como tampoco extrapola el fenómeno en relación con lo posible, para así llevar al lector hacia su propia reflexión.

La ausencia de una crítica social profunda o la presencia de una crítica sumamente superficial (pequeño-burguesa) corroboran ambas el ligamen de la así llamada CF costarricense, con lo establecido, con el status quo nomocrático-posmoderno.

Dentro del cambio del locus de alienación desde lo geográfico a lo cronológico, la CF costarricense muestra también sus particularidades. Para acceder a la idea de plantear un relato insertado en el futuro (lo posible) existe un abuso del recurso de datación, o sea, el actual escritor de CF costarricense cree que enunciando fechas alejadas del hoy, esto es recurso suficiente para implementar un cambio en la situación relacional de lo real y lo posible. En otras palabras, muchos de los escritores costarricenses legitimados como autores de CF colocan una fecha para verosimilizar su relato, pero ante la ausencia de la novedad en los ámbitos científicotecnológicos y en el respectivo lenguaje (los neologismos ya analizados) hacen de tal recurso un serio error.

De tal manera, se citan las fechas de 2010, 2020 y 2024 en "Objeto no identificado"; 2030 y 2060 en "Amor virtual"; 2020 en "Raquel y los emperadores"; 2082 en "El modelo defectuoso", etc. Sin embargo, la alteración en fechas (en lo cuantitativo) no produce un cambio cualitativo con respecto a la sociedad, como un todo, y a la $\mathrm{CF}$, en lo particular.

En otros casos, la acción se desarrolla en el pasado, como en los cuentos donde hay un viaje al pasado: "Sputnik", por ejemplo, donde la ocurrencia, como ya dijimos, suplanta a la ciencia y deslegitima así todo el relato como cuento de CF.

En la mayoría de casos faltan elementos para poder situar el locus cronológico, como en: "El impulso necesario para despertar", "Joyería mental", "Sofía", etc. También, algunos no son futuristas sino "pasadistas", como "Anti-Eureka" y "Los señores de piedra".

Dentro de la CF costarricense, el problema para plantear un locus cronológico sigue siendo la incapacidad de los escritores para manejar conocimientos de ciencias como la física, la astronomía, así como los fundamentos filosóficos y teóricos de las geometrías no-euclidianas, por ejemplo. Por tal motivo, estos escritores refieren constantemente a lo ya conocido, o sea, al pasado y al presente. 
Después de haber constatado que la creación de neologismos (propiamente dichos, en función de un novum) y el empleo de clichés a manera de neologismos es sumamente deficiente o inexistente en la CF costarricense analizada aquí, debido al poco o nulo conocimiento de los principios y leyes científicas, lo cual evita un adecuado desarrollo explicativo y cognoscitivo de la progresión del desarrollo tecnológico hacia futuro (lo cual sume a la CF costarricense en las ocurrencias); así como el maridaje de tal cuentística con el status quo y, por ende, la incapacidad de salirse del ámbito empírico de lo conocido; se puede determinar que no se representa dentro de la CF costarricense un mundo no completamente comprendido. De hecho, si no se presenta un mundo no completamente comprendido, entonces no hay novum. Por lo tanto, no existe el planteamiento de un novum dentro de la llamada CF costarricense, en general, ni en cada uno de los autores catalogados como escritores de CF, vistos separadamente, en particular.

Entonces, ¿por qué se legitima la producción literaria de estos autores (Iván MolinaJiménez, Daniel Garro, etc.) como CF? Este proceso de legitimación que hace pasar los cuentos de los autores costarricenses estudiados aquí, como representantes de la $\mathrm{CF}$, responde a un imperativo neoliberal. Es decir, recordemos que Friedman expresaba que la masa debe aprender a "leer, escribir y contar" (2010, p. 169); entonces la producción de baja calidad (técnicamente hablando) que hemos señalado en la CF costarricense va dirigida a cumplir con tal imperativo, of reciendo al lector joven una literatura simplista, acrítica, que busca solventar los problemas de lectura de las nuevas generaciones, con el anzuelo de la ciencia. Además, conlleva otro mandato de la pedagogía neoliberal (impulsada por el Banco Mundial), o sea, "la enseñanza de las ciencias y la matemática" (Malakolunthu, 2010, p. 80). Entonces, a la vez que se le da la mínima formación a un gran sector de los grupos no-dominantes, se inspira en algunos de ellos el gusto por la ciencia, la matemática y la tecnología; sin olvidar (recordemos los planteamientos de algunos cuentos de Iván Molina-Jiménez) que se les adoctrina a favor del pensamiento neoliberal. De esta manera, "la ciencia ficción ha logrado formar parte de los currículos de los high-schools y universidades anglosajonas y, poco a poco, se incorpora también al mundo docente de habla hispana" (Barceló-García, 2005, p. 4).

Así, se utiliza "la CF como recurso didáctico" (Petit-Pérez y Solbes-Matarredona, 2012 , p. 71), tratando de romper con "una imagen muy negativa de la ciencia a la que consideran (la mayoría de estudiantes) [paréntesis agregados] difícil, aburrida, sólo apta para genios y, por otra parte, la consideran la mayor responsable de algunos de los grandes problemas de nuestro tiempo" (Petit-Pérez y Solbes-Matarredona, 2012, p. 70).

Aunque Barceló-García (2005) y Petit-Pérez y Solbes-Matarredona (2012) refieren al ámbito europeo, la receta es la misma para nuestra realidad costarricense, dentro de un contexto que tiene dos vertientes de pensamiento, pero donde el desarrollo de la CF privilegia solo uno, el más reaccionario.

\section{Conclusión}

En lo que se ha denominado CF y de acuerdo con la perspectiva teórica suviniana que nos guía, en Costa Rica queda claro que no hay novum, luego la producción de Iván MolinaJiménez, Jessica Clark, Laura Casasa-[Núñez], Daniel Garro, etc., no es CF.

El amarre de los textos de estos autores, en cuanto fenómeno literario tardío de la Promoción de 1980, a la nomocracia posmoderna, impide, gnoseológica e ideológicamente, la producción de un novum que rompa con el status quo y que, a la vez, permita superar los 
serios fallos que tal grupo de escritores presenta no solo en el manejo de lo real y lo posible, sino también en el ámbito cognoscitivo de las leyes y principios de áreas como la física, las geometrías no-euclidianas, etc. ${ }^{5}$

El advenimiento de una CF bien definida a través de un novum, en Costa Rica, pasa por una renovación radical (tanto a nivel estilístico, como a nivel ideológico-cognoscitivo) de la Promoción de 1980. La CF necesita deslindarse del neoliberalismo, del giro lingüístico, etc., para surgir como un nuevo paradigma de escritura, que no esté al servicio de una pedagogía represiva, sino abierta a una profunda crítica social.

Es claro que el género de CF es la literatura más empírica que existe. Su ligamen con la tecnología y el aspecto práctico que esta conlleva le insertan a este género una base material que requiere de una visión del lenguaje que reconozca que "el lenguaje poético es un modo de aprehender la realidad" (Gaskin, 2013, p. 290) y, por consiguiente, permita a la CF trabajar "al modo de una crítica utópica para poner de relieve y desmitificar lo existente y, por ende, apuntar hacia alguna plenitud auténtica con la cual las privaciones de la realidad mundana son contrastadas" (Freedman, 2000, p. 82).

El futuro establecimiento de una $\mathrm{CF}$, definida en términos suvinianos, en Costa Rica, está en función de su desarrollo crítico y no como un simple mecanismo de control social (a través de la educación) o como un sacralizador del status quo, que ve la nomocracia posmoderna como una substancia, como una ontologización que se eterniza.

\section{Notas}

1. Esta y las siguientes traducciones de textos en inglés o francés son mías.

2. Evidentemente, "Una clase social educada en ciencias o al menos tecnológicamente adepta es una precondición para el desarrollo de una floreciente cultura de CF" (Csicsery-Ronay, 2012, p. 481).

3. Como indica Nealon, refiriéndose a lo que han seguido tales posiciones: "En breve, nosotros teóricos culturalistas y literarios somos, y hemos sido, neoliberales posmodernos" (2012, p. 174).

4 Estos cuentos se encuentran dispersos en los cuentarios: Posibles futuros (2009), Venus desciende: relatos de ciencia ficción (2009), Poe siglo XXI (2010), Objeto no identificado y otros cuentos de ciencia ficción (2011), Marte inesperado y otros relatos costarricenses de ciencia ficción (2012) y Lunas en vez de sombras y otros relatos de ciencia ficción (2013).

5 Podría pensarse con Moreno que a esta literatura costarricense le pasa lo que a la CF española, la cual "no está a menudo basada en las ciencias y no pretende romper con nuestra percepción de la realidad" (2011, p. 29). Esto debido a que "las características religiosas y políticas de España (compartidas por las antiguas colonias a través de la lengua) han influenciado este escape del materialismo y su consecuente repercusión literaria (sobre la CF)” (Moreno, 2011, pp. 29-30; paréntesis míos).

\section{Bibliografía}

Alfaro-Vargas, R. (2011). Capitalismo zombie. Contribución a la crítica del último capitalismo. Telos. Revista de Estudios Interdisciplinarios en Ciencias Sociales. 13 (3), 285-296.

Alfaro-Vargas, R. (2012). Henri Lefebvre y el fin de la nomocracia posmoderna. Revista de Ciencias Sociales. 3 (137), 105-121.

Antonio, R.J. (2012). After Neoliberalism. Wither Capitalism? Por G. Ritzer (Ed.). The WileyBlackwell Companion to Sociology. (567-587). Malden-Oxford: Wiley-Blacwell.

Apple, M.W. (2013). Can Education Change Society? Nueva York y Londres: Routledge. 
Barceló-García, M. (2005). Ciencia y ciencia ficción. Revista Digital Universitaria 6 (7): 1-10. Casasa-[Núñez], L. et ál. (2009). Posibles futuros. Cuentos de ciencia ficción. San José: EUNED. Castillo-[Rojas], M. et ál. (2011). Objeto no identificado y otros cuentos de ciencia ficción. San José: EUNED.

Chamu, A. et ál. (2010). Poe siglo XXI. Ciencia ficción costarricense. San José: Editorial Clubdelibros.

Chaves, J.R. et ál. (2013). Lunas en vez de sombras y otros relatos de ciencia ficción. San José: EUNED.

Chibber, V. (2013). Postcolonial Theory and the Specter of Capital. Londres: Verso.

Cramer, K. (2003). Hard science fiction. Por E. James y F. Mendlesohn (Eds.). The Cambridge Companion to Science Fiction. (186-196). Nueva York: Cambridge University Press.

Csicsery-Ronay, I.J. (2003). Marxist theory and science fiction. Por E. James y F. Mendlesohn (Eds.). The Cambridge Companion to Science Fiction. (113-124). Nueva York: Cambridge University Press.

Csicsery-Ronay, I.J. (2012). What Do We Mean When We Say "Global Science Fiction"? Reflections on a New Nexus. Science Fiction Studies. 39 (3), 478-494.

Fraser, N. (2013). Fortunes of Feminism: From State-Managed Capitalism to Neoliberal Crisis. Londres y Nueva York: Verso.

Freedman, C. (2000). Science Fiction and Utopia: A Historico-Philosophical Overview. Por P. Parrinder (Ed.). Learning from Other Worlds. Estrangement, Cognition and the Politics of Science Fiction and Utopia. (72-97). Liverpool: Liverpool University Press.

Friedman, M. (2010). Capitalisme et liberté. París: Leduc.s Éditions.

Gaskin, R. (2013). Language, Truth, and Literature. A Defense of Literary Humanism. Oxford: Oxford University Press.

Haywood-Ferreira, R. (2011). Presentación. Por M. Castillo et ál. Objeto no identificado y otros cuentos de ciencia ficción. (vii-xiii). San José: EUNED.

James, E. (2000). Before the Novum: The Prehistory of Science Fiction Criticism. Por P. Parrinder (Ed.). Learning from Other Worlds. Estrangement, Cognition and the Politics of Science Fiction and Utopia. (19-35). Liverpool: Liverpool University Press.

Jara, L.E. (2010). El legado del cuervo. Por A. Chamu et ál. Poe siglo XXI. Ciencia ficción costarricense. San José: Editorial Clubdelibros.

Klein, G. (2000). From the Images of Science to Science Fiction. Por P. Parrinder (Ed.). Learning from Other Worlds. Estrangement, Cognition and the Politics of Science Fiction and Utopia. (119-126). Liverpool: Liverpool University Press.

Malakolunthu, S. (2010). Implementation of Education Reform Policies: The Issue of Structural and Cultural Discordance. Por J. Zajda (Ed.). Globalisation, Ideology and Education Policy Reforms. (79-85). Dordrecht, Heidelberg, Londres, New York: Springer.

Molina-Jiménez, I. (2009). Venus desciende: relatos de ciencia ficción. Alajuela: Autor. 
Molina-Jiménez, I. (2010). Género en construcción: la ciencia ficción costarricense. A propósito de la novela El vuelo del Ra, de Manuel Delgado. A Contracorriente. 8 (1), 408-415.

Molina-Jiménez, I. et ál. (2012). Marte inesperado y otros relatos costarricenses de ciencia ficción. San José: Grupo Nación.

Moreno, F.Á. (2006). La ficción prospectiva: propuesta para una delimitación del género de la ciencia ficción”. Por T. López-Pellisa y F.Á. Moreno (Eds.). Ensayos sobre ciencia ficción y literatura fantástica. (65-93). Madrid: Asociación Cultural Xatafi y Universidad Carlos III de Madrid.

Moreno, F.Á. (2011). Recent Spanish Science Fiction and its Modes. SFRA Review. (297), 29-32.

Moylan, T. (2000). 'Look into the dark': On Dystopia and the Novum. Por P. Parrinder (Ed.). Learning from Other Worlds. Estrangement, Cognition and the Politics of Science Fiction and Utopia. (51-71). Liverpool: Liverpool University Press.

Nealon, J.T. (2012). Post-postmodernism or, The Cultural Logic of Just-in-Time Capitalism. Standford, CA: Standford University Press.

Parrinder, P. (2000). Introduction: Learning from Other Worlds. En Learning from Other Worlds. Estrangement, Cognition and the Politics of Science Fiction and Utopia. (1-16). Liverpool: Liverpool University Press.

Petit-Pérez, M.F. y Solbes-Matarredona, J. (2012). La ciencia ficción y la enseñanza de las ciencias. Enseñanza de las ciencias. Revista de investigación y experiencias didácticas. 30 (2), 69-86.

Quesada-Soto, Á. (2000). La narrativa costarricense del último tercio de siglo. Letras. (32), $17-43$.

Ríos-Quesada, V. (2012). Prólogo. Por I. Molina-Jiménez et ál. Marte inesperado y otros relatos costarricenses de ciencia ficción. (7-9). San José: Grupo Nación.

Roberts, A. (2006a). Science Fiction. (2 ${ }^{\text {da }}$ ed.). Londres y Nueva York: Routledge.

Roberts, A. (2006b). The History of Science Fiction. Nueva York: Palgrave Macmillan.

Seed, D. (2011). Science Fiction. A Very Short Introduction. Nueva York: Oxford University Press.

Shippey, T. (2005). Hard Reading: The Challenges of Science Fiction. Por D. Seed (Ed.). A Companion to Science Fiction. (11-26). Malden-Oxford-Victoria: Blackwell.

Slusser, G. (2005). The Origins of Science Fiction. Por D. Seed (Ed.). A Companion to Science Fiction. (27-42). Malden-Oxford-Victoria: Blackwell.

Suvin, D. (1979). Metamorphoses of Science Fiction. On the Poetics and History of a Literary Genre. New Haven y Londres: Yale University Press.

Suvin, D. (2000). Afterword: With Sober, Estranged Eyes. Por P. Parrinder (Ed.). Learning from Other Worlds. Estrangement, Cognition and the Politics of Science Fiction and Utopia. (233-271). Liverpool: Liverpool University Press.

Westfahl, G. (2005). Hard Science Fiction. Por D. Seed (Ed.). A Companion to Science Fiction. (187-201). Malden-Oxford-Victoria: Blackwell. 